astro-ph/0308403

CERN-TH/2003-197

FTUV-030822

\title{
Synchrotron Radiation from the Crab Nebula Discriminates between Models of Space-Time Foam
}

\author{
J. Ellis ${ }^{a}$, N.E. Mavromatos ${ }^{b, c}$ and A.S. Sakharov ${ }^{a, d}$ \\ ${ }^{a}$ CERN, Theory Division, CH-1211 Geneva 23, Switzerland. \\ ${ }^{b}$ Department of Physics, King's College London, University of London, Strand, \\ London WC2R 2LS, U.K. \\ c Departamento de Física Téorica, Universidad de Valencia, E-46100, Burjassot, \\ Valencia, Spain. \\ ${ }^{d}$ Swiss Institute of Technology, ETH-Zürich, 8093 Zürich, Switzerland.
}

\begin{abstract}
It has been argued by Jacobson, Liberati and Mattingly that synchrotron radiation from the Crab Nebula imposes a stringent constraint on any modification of the dispersion relations of the electron that might be induced by quantum gravity. We supplement their analysis by deriving the spectrum of synchrotron radiation from the coupling of an electrically-charged particle to an external magnetic fields in the presence of quantum-gravity effects of the general form $\left(E / M_{Q G}\right)^{\alpha}$. We find that the synchrotron constraint from the Crab Nebula practically excludes $\alpha \lesssim 1.74$ for $M_{Q G} \sim m_{P}=1.2 \times 10^{19} \mathrm{GeV}$. On the other hand, this analysis does not constrain any modification of the dispersion relation of the photon that might be induced by quantum gravity. We point out that such quantum-gravity effects need not obey the equivalence principle, a point exemplified by the Liouville-string D-particle model of space-time foam. This model suggests a linear modification of the dispersion relation for the photon, but not for the electron, and hence is compatible with known constraints from the Crab Nebula and elsewhere.
\end{abstract}

CERN-TH/2003-197

August 2003 


\section{Introduction}

Modified dispersion relations have been first suggested in the context of stringy quantum gravity (QG) in [1], based on the Liouville string approach to quantum space time 2]. Later, analogous modifications have also been suggested in the contexts of other models of QG, either phenomenological [3] or theoretical. Examples of the latter include loop gravity [4] and novel models in which the Planck scale is viewed as a length that remains invariant under modified non-linear Lorentz transformations [5]. Violations of Lorentz symmetry had been suggested earlier [6] as a way of avoiding the Greisen-Zatsepin-Kuzmin (GZK) cutoff for ultra-high-energy cosmic rays (UHECRs).

Liouville-string models of space-time foam [2] motivate corrections to the usual relativistic dispersion relations that are of first order in the particle energies, corresponding to a vacuum refractive index $\eta \simeq 1-\left(E / M_{Q G}\right)^{\alpha}: \alpha=1$. These effects are associated generically with deviations from conformal invariance in the effective theory of low-energy excitations interacting with singular or topologically nontrivial quantum-gravitational (QG) degrees of freedom, inaccessible to low-energy observers [2]. Models with quadratic dependences of the vacuum refractive index on energy: $\alpha=2$ have also been considered [7].

The phenomenology of such models has grown rapidly. Following the original suggestion [8, 9] to place bounds on the effective QG scale by comparing the arrival times of photons with different energies from gamma-ray bursters (GRBs), in order to probe the refractive index that might be induced by $\mathrm{QG}$, it has also been pointed out that electrically-charged fermionic probes, either in astrophysics [10] or in terrestrial atomic and nuclear physics experiments [11, 12, 13], can constrain severely phenomenological models of space-time foam with modified dispersion relations.

The most severe of all the known constraints seems to be that associated with synchrotron radiation from the Crab Nebula, whose sensitivity exceeds the Planck scale by (at least) seven orders of magnitude [12, in the case of a linear modification of the dispersion relation for the electron. For photons, on the other hand, the most precise probe of a possible QG-induced refractive index comes from an analysis of 
arrival times of emissions from GRBs [9, which impose $M_{Q G} \gtrsim 10^{16} \mathrm{GeV}$. Analyses of light from active galactic nuclei (AGNs) and pulsars [14] also reveal no evidence of QG effects, and may have comparable sensitivities to $M_{Q G}$ for photons.

In the first part of this paper, we revisit the analysis of [12, by considering in detail the propagation of a charged matter probe interacting with an external magnetic field in the case of a generic modified dispersion relation, not necessarily linearly suppressed by the Planck scale. Our analytical result includes explicitly corrections due to the calculable change in the synchrotron radius in the presence of such QG effects. We confirm that the synchrotron constraint provided by the Crab Nebula [12] on the electron's dispersion relation is robust theoretically. Unless it is relaxed for astrophysical reasons, this type of constraint is so strong that it already excludes an $\alpha \leq 1.7$ correction to the vacuum refractive index for electrons, and has the potential to be sensitive to a quadratic correction: $\alpha=2$ in the near future, when higher-precision data become available.

However, this does not mean that all types of QG corrections with $\alpha \leq 2$ are excluded. The escape route is for QG to violate the equivalence principle, so that $\alpha=1$ and $\eta<1$ for photons, whilst $\eta=1$ for electrons. Remarkably, this is exactly what happens in the Liouville model of space-time foam proposed in [15], in the modern framework of the D-brane approach to QG [16]. The reason for this violation of the equivalence principle for different categories of particles is explained briefly at the end of this paper, and is described in more detail elsewhere [17.

The structure of the article is as follows: in the next Section we summarize the Crab Nebula analysis of [12. Then, in Section 3 we present an analytical derivation of the synchrotron radiation spectrum in the presence of modified dispersion relations, including the change in the synchrotron radius that we show to be a small effect. We also show that a quadratic modification of the electron's dispersion relation with $M_{Q G}=m_{P}$ is marginally excluded by the Crab data. Finally, in Section 4, we discuss the ability of this constraint to exclude some models of QG, showing how the specific model of space-time foam in [15] evades it by violating the equivalence principle in a characteristic way. 


\section{The Synchrotron Radiation Constraint from the Crab Nebula}

Let us briefly summarize the main points of [12, who first proposed considering the constraints implied by synchrotron radiation from the Crab Nebula. We highlight some steps which were not made explicit in their analysis, motivating our derivation in the next Section of an analytical mathematical description of synchrotron radiation in the presence of modified dispersion relations.

Following [12], we assume the modified dispersion relations (in units of the speed of light in vacuo $c$, which we now set to unity)

$$
\begin{aligned}
\omega^{2}(k) & =k^{2}+\xi_{\gamma} \frac{k^{3}}{M_{P}}, \\
E^{2}(p) & =m_{0}^{2}+p^{2}+\xi_{e} \frac{p^{3}}{M_{P}},
\end{aligned}
$$

for photons and electrons, respectively, where $\omega$ and $k$ are the photon frequency and wave number, and $E$ and $p$ are the electron energy and momentum, with $m_{0}$ the electron rest mass. Here we assume linear QG effects, characterized by parameters $\xi_{\gamma}$ and $\xi_{e}$, extracting the Planck mass scale $m_{P}=1.22 \times 10^{19} \mathrm{GeV}$. We also assume here that energy and momentum are conserved in particle interactions ${ }^{1}$. We expect that the parameters $\xi_{\gamma}$ and $\xi_{e}$ are negative semi-definite, given that in the framework of Liouville-inspired quantum gravity [15] there are at most subluminal modifications, if any, and superluminal modifications would cause troubles with gravitational Čerenkov radiation [20. We note that the modifications to the dispersion relation proposed in [15] arise from a non-Minkowski induced metric in target space, which leads to some formal differences from the approach of [12, as we comment later.

We now recapitulate the derivation of constraints from the Crab Nebula synchrotron radiation on phenomenological models of quantum gravity that incorporate (2) for electrons. It was assumed in [12] that the usual description of synchrotron

\footnotetext{
${ }^{1}$ This may be questioned [18, 19], but any plausible violation is unimportant for our purposes.
} 
radiation was applicable, and the following facts about spectrum of the Crab nebula were used:

- Synchrotron emission is observed up to energies of about $0.5 \mathrm{GeV}$, where the inverse Compton hump begins to dominate the spectrum.

- Photons with energies up to $50 \mathrm{TeV}$ are observed.

- Electrons with energies up to at least $50 \mathrm{TeV}$ are required by energy conservation to produce the observed flux of $50 \mathrm{TeV}$ photons by inverse Compton scattering.

In the standard Lorentz-invariant theory, the $0.5 \mathrm{GeV}$ synchrotron emission in the magnetic field of the Crab nebula $(\sim 0.3 \mathrm{mG})$ is generated by electrons of energy $5 \times 10^{4} \mathrm{TeV}$. The inference of this energy assumes, however, Lorentz invariance, which is just what we want to test. Hence the authors of [12] adopted the more conservative lower value of $50 \mathrm{TeV}$, which is inferred using only energy conservation in the inverse Compton process.

In standard electrodynamics, accelerated electrons in a magnetic field emit synchrotron radiation with a spectrum that cuts off sharply at a frequency $\omega_{c}$ which can be calculated as follows. According to the standard theory, electrons in an external magnetic field $H$, follow helical orbits transverse to the direction of $H$, with the orbit radius $R$ given by

$$
R=\frac{E}{H}
$$

where $E$ is the total energy of the electron. The orbital frequency of the electron in this case is:

$$
\omega_{0}=\frac{\beta_{\perp}}{R}
$$

where $\beta_{\perp} \equiv v_{\perp}$ is the component of the velocity of the electron perpendicular to the direction of the magnetic field. It was argued in [21] that there could be modifications to $R$ that might affect the results of [12]. We show later that there are indeed QG induced modifications, but that these do not change the central result of [12]. 
We recall that, in the standard Lorentz-invariant (LI) theory of synchrotron radiation, an electron moving in a magnetic field $H$ emits a discrete spectrum of frequencies, which are integer multiples of:

$$
\omega_{0}^{*}=\frac{\omega_{0}}{\sin ^{2} \theta},
$$

where $\theta$ is the angle between the velocity of the electron, $\vec{v}$, and the direction of $\vec{H}$. The spectrum of the emitted radiation has a maximum at a critical frequency

$$
\omega_{c}^{L I}=\frac{3}{2} \frac{e H}{m_{0}} \frac{1}{1-\beta^{2}},
$$

where $e$ is the electron charge. The superfix $L I$ in (66) stresses that this formula is based on a LI approach, in which one calculates the electron trajectory in a given magnetic field $H$ and the radiation produced by a given current, using the relativistic relation between energy and velocity. All of these assumptions could in principle be affected by violations of Lorentz symmetry.

It was stated in 12 that, even without assuming Lorentz invariance, the critical frequency is given by

$$
\omega_{c}=\frac{3}{4} \frac{1}{R \delta(E)} \frac{1}{c\left(\omega_{c}\right)-v(E)}
$$

where $\delta(E)$ is the opening angle for the forward-directed radiation pattern, and $c\left(\omega_{c}\right)$ and $v(E)$ are the group velocities of the radiation and electron respectively. The self-consistent solution of (77) for $\omega_{c}(E)$ determines the cutoff synchrotron frequency. There was no attempt to solve this equation in [12]. Instead, it was argued that one can replace $c\left(\omega_{c}\right)$ by $c=1$, so that the self-consistent solution is simply equal to the right-hand side of (7). To motivate this result, one may note that the electron and photon speeds are very close to the low-energy speed of light in vacuo $c=1$.

The synchrotron radius $R$ for any given energy is determined by the equation of motion of the electron. The authors of [12] assumed that gauge invariance is preserved, and used the usual minimal coupling. To find the electron equation of motion in a magnetic field $H$, they used the dispersion relation (2) for the Hamiltonian, with the momentum replaced by

$$
\mathbf{p} \rightarrow \mathbf{p}-e \mathbf{A}
$$


where $\mathbf{A}$ is a vector potential for the magnetic field. This yields the equation of motion $\mathbf{a}=\left[1+3 \xi_{e} E / 2 M\right](e / E) \mathbf{v} \times \mathbf{H}$, where only the term of lowest order in $\xi_{e}$ is kept, when one assumes $E \gg m_{0}{ }^{2}$. Since $E \ll M$, the authors of [12] argued that Lorentz violation would make very little difference to the orbital equation, so that the radius $R$ would be related to the magnetic field $H$ and the energy $E$ of the electron by the standard formula (3). These arguments have been refuted qualitatively in [21], with a subsequent reply in [22]. In order to arrive at a decisive conclusion, we embark in this article on a detailed quantitative analysis of the QG modification of the curvature radius.

In the next Section, we revisit the analysis of [12, presenting a derivation that includes the modification of this formula for the orbit radius due to the modification of the dispersion relation. The potential importance of this modification has been stressed in 21, but we find it to be relatively small. Our calculations should be accurate for all energies $E \lesssim 0.3 M_{P} /\left|\xi_{e}\right|$. The analysis of [12] can then be used to infer a strong lower bound of the effective quantum gravity scale for electrons: $M_{P} /\left|\xi_{e}\right| \sim 10^{27} \mathrm{GeV}$. However, we reiterate that there are no such strict bounds for photons, for which the most stringent bounds on the quantum gravity scale $M_{P} /\left|\xi_{\gamma}\right| \gtrsim 10^{16} \mathrm{GeV}$ have been derived from gamma-ray bursts [9].

One might be tempted to assume universality for the QG corrections $\xi_{\gamma}$ and $\xi_{e}$, as a consequence of the equivalence principle, in which case the electron bound would exclude observable linear QG modifications of dispersion relations in general. However, one should not exclude a priori the possibility that the equivalence principle might be violated for such QG corrections, with different effective quantum scales for different particle species, as considered in [6, 23]. We argue in Section 5 that this is indeed the case in the Liouville model for space-time foam of [15], where electric charge conservation makes the foam medium transparent to charged probes such as electrons, but not to photons.

Before proceeding with our detailed derivation of the modified synchrotron spec-

\footnotetext{
${ }^{2}$ We note that the covariantization (8) was done assuming that the background space-time is flat Minkowski space, as in the phenomenological analysis of [12].
} 
trum in the next Section, we first recall how (3) was used in [12] to derive synchrotron radiation bounds. Using the dispersion relations (112), and keeping terms suppressed only linearly by the QG scale $M_{P}$, one may write the difference of group velocities in the denominator of the last term of (7) as:

$$
c(\omega)-v(E)=\xi_{\gamma} \omega+\left(m_{0}^{2} / 2 E^{2}\right)-\xi_{e} E,
$$

The synchrotron radiation constraint comes from maximizing the electron group velocity with respect to the energy $E$, which yields for the maximum photon frequency [12]:

$$
\omega_{c}=\frac{3}{2} \frac{e H}{m_{0}} \frac{m_{0} \gamma(E)}{E} \gamma^{2}(E) .
$$

Comparing (10) with (6), it was observed in [12] that the factor $m_{0} \gamma(E) / E$, which is different from unity as a consequence of Lorentz violation, is a bounded function of $E$,

$$
\gamma(E)=\left(1-v^{2}\right)^{-1 / 2} \approx\left(\frac{m_{0}^{2}}{E^{2}}-2 \xi_{e} \frac{E}{M}\right)^{-1 / 2} .
$$

Maximizing $\omega_{c}$ with respect to the energy $E$ yields

$$
\omega_{c}^{\max }=0.34 \frac{e H}{m_{0}}\left(-\xi_{e} m_{0} / M\right)^{-2 / 3},
$$

which is attained at the energy $E_{\max }=\left(-2 m_{0}^{2} M / 5 \xi_{e}\right)^{1 / 3}=10\left(-\xi_{e}\right)^{-1 / 3} \mathrm{TeV}$. The frequency $\omega_{c}^{\max }$ is the highest possible value of the critical synchrotron frequency for any electron energy. The rapid decay of synchrotron emission at frequencies larger than $\omega_{c}$ implies that most of the flux at a given frequency in the synchrotron radiation peak observed coming from the Crab Nebula is due to electrons for which $\omega_{c}$ is above that frequency. Thus $\omega_{c}^{\max }$ must be greater than the maximum observed synchrotron emission frequency $\hbar \omega_{\text {obs }}$, which yields the constraint

$$
\xi_{e}>-\frac{M}{m_{0}}\left(\frac{0.34 e H}{m_{0} \omega_{\mathrm{obs}}}\right)^{3 / 2} .
$$

This was the main result of [12. It implies, when one takes into account indicative physical values/estimates of the various quantities entering (13), a very small upper 
bound on the magnitude of the coefficient $\left|\xi_{e}\right|$ of the QG modification of the electron dispersion relation (which is assumed to be negative, corresponding to subluminal propagation):

$$
\left|\xi_{e}\right|<7 \times 10^{-8} .
$$

As already mentioned, this analysis does not yield a stringent constraint on the magnitude of the photon coefficient $\left|\xi_{\gamma}\right|$.

At this stage, we remark that, in the framework of our Liouville model of spacetime foam, we have derived QG modifications only for massless neutral particles such as the photon and photino, the latter being used as a model for the neutrino [24]. As discussed later in this paper, different categories of particles may have different QG modifications in their dispersion relations, and these derivations do not apply to electrons.

\section{Analytic Derivation of the Synchrotron Radia- tion Constraint}

We now present an analytical derivation of the QG modification to the synchrotronradiation formula, for generic subluminal modifications of the electron dispersion relation of the form:

$$
E^{2}(p)=m_{0}^{2}+p^{2}-\frac{p^{2+\alpha}}{\mathcal{M}^{\alpha}},
$$

where $\mathcal{M}\left(=M_{P} /\left|\xi_{e}\right|\right.$ for $\left.\alpha=1\right)$ sets the effective QG scale in the modifications of the dispersion relation for the electron, which we seek to bound by means of the synchrotron radiation. We use the modified dispersion relation (15) for the Hamiltonian of a charged spinless particle probe, which we place in an external magnetic field. It is convenient to parametrize the QG effects $p^{2+\alpha} / \mathcal{M}^{\alpha}$ by means of the refractive index in vacuo $\eta$ :

$$
\eta=1-\left(\frac{E}{\mathcal{M}}\right)^{\alpha}, \quad \alpha \geq 1 .
$$


At ultra-relativistic energies, one may replace $p^{\alpha}$ by $E^{\alpha}$ in the dispersion relation, and thus use the following for the Hamiltonian:

$$
E^{2}=p^{2}+m_{0}^{2}-p^{2}\left(\frac{E}{\mathcal{M}}\right)^{\alpha} .
$$

Notice that, in writing the dispersion relation in the form (17), we have ignored terms proportional to the mass $m_{0}$ in the QG modifications, expecting them to be subleading, which is a self-consistent approximation at ultra-relativistic electron energies, as we shall see. Moreover, for the range of energies relevant to our problem, we can also ignore terms of order $(E / \mathcal{M})^{2 \alpha}$ or higher in the following.

Before analyzing the consequences of (17), we first make some important remarks about the Liouville foam model of [15], as opposed to generic models of phenomenological modified dispersion relations in flat space-times, such were considered in [12]. One important difference of the Liouville model of foam is that the modifications in the dispersion relation of a string probe owe their existence in the appearance of a non-trivial induced target-space metric:

$$
G_{00}=-1 ; \quad G_{i j}=\delta_{i j} ; \quad G_{0 i}=g_{s} \Delta p_{i} / M_{s} \sim \frac{1}{2} g_{s} p_{i} / M_{s}
$$

where $M_{s} / g_{s}=\mathcal{M}$ is the effective QG scale, $M_{s}$ is the string scale, $g_{s}$ a string coupling, and $\Delta p_{i}$ is the momentum transfer during the scattering of the string with the D-particle defect [15. A generic dispersion relation for a particle with mass $m_{0}$ in the metric background (18) is:

$$
p_{\mu} p_{\nu} G^{\mu \nu}=-m_{0}^{2}
$$

which, as can readily be seen, implies [15] a modified dispersion relation of subluminal form (17), in the case where the direction of recoil of the D-particle defect is opposite to that of the incident particle, in the frame where the (massive) defect was initially at rest,

$$
\vec{p} \cdot u^{i} \sim \frac{\vec{p} \cdot \vec{p}}{2 \mathcal{M}}<0 .
$$

We recall that the requirement of subluminality emerges from specific properties of the underlying string theory, namely the Born-Infeld form of the effective action describing the dynamics of the excitations of the recoiling D-particle [15]. 
Physically, this opposite recoil corresponds to capture of the particle probe with subsequent decay of the defect, and emission of a particle with modified momentum ${ }^{3}$. It is this feature that may lead to violations of the equivalence principle, if electrons and photons have different probabilities for this process. This indeed happens in simple models, as we discuss later.

We also observe from (19) that the inverse of the metric (18) results in a higherorder 'renormalization' of the rest-mass term $m_{0}^{2}$ in the dispersion relation (17): $m_{0}^{2} \rightarrow m_{0}^{2}\left(1+|\vec{u}|^{2}\right)$. We ignore such higher-order effects here, which is a selfconsistent approximation for the range of energies relevant to the problem.

In what follows, we keep our analysis as generic as possible, so as to minimize the dependence on specific models of foam. To this end, we use the modified dispersion relation (17) to define the momentum operator in a way that takes properly into account the QG modification ${ }^{4}: p \rightarrow \tilde{p} \equiv p \eta$, which becomes $\tilde{p}=p\left(1-\frac{E}{\mathcal{M}}\right)$ in the linear case, where the minus sign indicates subluminal propagation. This definition implies that we define an 'effective' momentum squared as the part one has to subtract from the square of the energy in order to obtain the rest mass squared ${ }^{5}$.

It is this $\tilde{p}$ that we shall couple to the external electromagnetic field, $\vec{A}$, ensuring gauge invariance by minimal substitution, which implies the electromagnetic covariantization

$$
\tilde{\mathbf{p}} \rightarrow \tilde{\mathbf{p}}+\frac{e_{0}}{c} \overrightarrow{\mathbf{A}}
$$

As we now show in detail, in the case of QG-induced modifications of the electron and

\footnotetext{
${ }^{3}$ Ref. [21 considered synchrotron radiation in terms of an interaction $e^{-}+\gamma_{H} \rightarrow e^{-}+\gamma$, where $\gamma_{H}$ denotes a soft photon representing the magnetic field. In our approach, we consider the quantum theory of synchrotron radiation [25], in which an electron in a classical magnetic field emits a quantum of synchrotron radiation by changing the state of its wave function. This emission depends on a three-particle vertex that has been discussed in the context of our model of quantum gravity in [19, and could in principle violate energy conservation. However, such effects are important only when all three of the quantum particles have large momenta, which is not the case here, since the emitted photon is relatively soft.

${ }^{4}$ In the Liouville foam model [15], this definition could be thought of as the gravitational covariant derivative associated with the non-Minkowski induced metric (18).

${ }^{5}$ This definition resembles that in general relativity, where one defines the effective potential in a curved Schwarzschild space-time as the part one has to subtract from the square of the energy in order to obtain the square of the radial kinetic energy term.
} 
photon dispersion relations, the maximal synchrotron frequency (6) gets modified, because of the modification of the orbital frequency as well as the velocity factor $\beta$ of the electron:

$$
\omega_{1}=\frac{\omega_{0}^{Q G}}{1-\beta_{Q G}},
$$

where we now concentrate on the longitudinal motion.

The modification of the orbital frequency is connected with QG effects on the radius of the orbit, due to the back-reaction of the foamy QG medium on the propagation of the electron. Such effects are purely quantum in nature, and therefore require a quantum treatment of the motion of an electron of charge $e=-e_{0}$ in a magnetic field, which we shall now present. For our purposes of estimating the change in the radius of the orbit as a result of the QG modifications of the dispersion relation, we do not take into account the spin of the electron, but consider instead the simpler problem of a spinless particle in a magnetic field. We justify the validity of this simplification later in the article.

We consider a magnetic field along the $z$ axis, which implies a vector potential $A_{x}=-\frac{1}{2} y H, \quad A_{y}=\frac{1}{2} x H, \quad A_{z}=0$, and use the QG-induced dispersion relation (17) for the Hamiltonian operator of the quantum mechanical problem that describes the coupling of a spinless particle with this potential. The associated Klein-Gordon equation [25] can then be written in the following form (here we state explicitly the dependences on the speed of light in vacuo $c$ ):

$$
\left\{E^{2}-c^{2}\left(-\vec{p} \eta+\frac{e_{0}}{c} \vec{A}\right)^{2}-m_{0}^{2} c^{4}\right\} \Psi(\vec{r}, t)=0, \quad \vec{p} \equiv-i \hbar \vec{\nabla}
$$

where $\eta$ is defined in (16). Proceeding, as usual, to the stationary case via $\psi(\vec{r}, t)=\exp \left(-i \frac{E}{\hbar} t\right) \psi(\vec{r})$, we have:

$$
\left(k^{2}-k_{0}^{2}+\eta^{2} \nabla^{2}-\eta \frac{2 i e_{0}}{c \hbar} \vec{A} \cdot \vec{\nabla}-\frac{e_{0}^{2}}{c^{2} \hbar^{2}} A^{2}\right) \psi(\vec{r})=0,
$$

where $k=E / c \hbar$ and $k_{0}=m_{0} c / \hbar$. In what follows, we consider only positive values of the energy, i.e., $k>0$, in which case we may rewrite (24) in terms of cylindrical 
coordinates $(r, \varphi, z)$, as follows:

$$
\left(k^{2}-k_{0}^{2}+\eta^{2} \nabla_{r}^{2}+\eta^{2} \frac{\partial^{2}}{\partial z^{2}}+\frac{\eta^{2}}{r^{2}} \frac{\partial^{2}}{\partial \varphi^{2}}-2 i \eta \gamma \frac{\partial}{\partial \varphi}-\gamma^{2} \eta^{2}\right) \psi(r, \varphi, z)=0
$$

where $\gamma \equiv e_{0} H / 2 c \hbar$.

Following [12, we now assume that the $z$ component of momentum and the angular momentum are conserved as a consequence of rotational symmetry, which is maintained in the analysis of [12] ${ }^{6}$. Changing variables to $\varphi_{1}=\varphi / \eta$ and $\tilde{z}=z / \eta$, we make the ansatz:

$$
\psi=\frac{e^{i \ell \varphi_{1}} e^{i k_{3} \tilde{z}}}{(2 \pi)^{1 / 2} L^{1 / 2}} f(r)
$$

where $k_{3}=2 \pi n_{3} / L$, and $\ell$ and $n_{3}$ are the azimuthal and vertical quantum numbers (corresponding to the appropriate components of the orbital angular momentum), which may take on positive and negative values, including zero. The radial part of the wave-function satisfies:

$$
\left\{\rho \frac{d^{2}}{d \rho^{2}}+\frac{d}{d \rho}+\lambda-\frac{\ell}{2}-\frac{\eta^{2} \rho}{4}-\frac{\ell^{2}}{4 \rho \eta^{2}}\right\} f=0,
$$

where $\rho \equiv \gamma r^{2} / \eta^{2}$, and $\lambda=\left(k^{2}-k_{0}^{2}-k_{3}^{2}\right) / 4 \gamma$.

The asymptotic behaviour as $\rho \rightarrow \infty$ of the radial function $f(\rho)$ can be chosen such that

$$
f_{\infty}=f(\infty) \sim e^{-\frac{\rho}{2}}, \quad f_{0}=f(0) \sim p^{\ell / 2} .
$$

This allows us to write

$$
f=f_{\infty} f_{0} u(\rho)=e^{-(\rho / 2)} p^{\ell / 2} u(\rho),
$$

where $u(\rho)$ satisfies the equation

$$
\rho u^{\prime \prime}+(\ell+1-\rho) u^{\prime}+\left(\lambda-\ell-\frac{1}{2}+\frac{\rho}{4}\left(1-\eta^{2}\right)+\frac{\ell^{2}}{4 \rho}\left(1-\frac{1}{\eta^{2}}\right)\right) u=0 .
$$

\footnotetext{
${ }^{6}$ In certain models of anisotropic QG foam, angular momentum is not necessarily conserved. In such a case, our analysis here should be modified.
} 
We now write this equation in a form that can be compared with the confluent hypergeometric equation

$$
\rho u^{\prime \prime}+(\kappa-\rho) u^{\prime}-\left(a+a_{1} \rho+\frac{a_{2}}{\rho}\right) u=0,
$$

where we set $\kappa=\ell+1, a=-\lambda+\ell+\frac{\ell}{2}, a_{1}=-\frac{1-\eta^{2}}{4}$ and $a_{2}=\frac{\ell^{2}}{4}\left(1-\frac{1}{\eta^{2}}\right)$. Making the substitution $u=g \rho^{-\kappa / 2} e^{\rho / 2}$ and changing the variable $\rho=\frac{\tilde{\rho}}{\sqrt{4 a_{1}+1}}$ in (31), we obtain

$$
g^{\prime \prime}+\left[-\frac{1}{4}+\frac{\frac{\kappa}{2}-a}{\tilde{\rho} \sqrt{4 a_{1}+1}}+\frac{\frac{\kappa}{2}-\frac{\kappa^{2}}{4}-a_{2}}{\tilde{\rho}^{2}}\right] g=0 .
$$

Substituting $\bar{k}=\left(\frac{\kappa}{2}-a\right) / \sqrt{4 a_{1}+1}, m=\sqrt{\frac{1}{4}-\frac{\kappa}{2}+\frac{\kappa^{2}}{4}+a^{2}}$, we reduce (32) to the form of a standard Whittaker equation,

$$
g^{\prime \prime}+\left[-\frac{1}{4}+\frac{\bar{k}}{\tilde{\rho}}+\frac{\frac{1}{4}-m^{2}}{\tilde{\rho}^{2}}\right] g=0 .
$$

The solution for positive energies is

$$
g=\tilde{\rho}^{m+1 / 2} e^{-\tilde{\rho} / 2}{ }_{1} F_{1}\left(m+\frac{1}{2}-\bar{k} ; 2 m+1 ; \tilde{\rho}\right),
$$

where ${ }_{1} F_{1}(A, B, \tilde{\rho})$ is the confluent hypergeometric function, which grows exponentially as $\rho \rightarrow \infty$. Therefore, the asymptotic behavior of the solution of (130) for large $\rho$ takes the form

$$
u \simeq e^{\rho} \rho^{1 / 2-\lambda} \frac{\Gamma(B)}{\Gamma(A)},
$$

where $\Gamma(x)$ is the standard Gamma function. This implies that, in order to respect the finiteness of the probability of the wave-function as $\rho \rightarrow \infty$, one has to impose the vanishing of the factor $1 / \Gamma\left(m+\frac{1}{2}-\bar{k}\right)$, i.e., the selection rule:

$$
m+\frac{1}{2}-\bar{k}=-s, \quad s=0,1,2, \ldots
$$

where $s$ is the well-known radial quantum number. Substituting the value of $\lambda$ given above, c.f., (27), we obtain:

$$
\frac{k^{2}-k_{0}^{2}-k_{3}^{2}}{4 \gamma}=\frac{1}{2} \ell\left(\frac{\left(2 \eta^{2}-1\right)^{1 / 2}}{\eta}+1\right)+s \eta+\frac{\eta}{2} .
$$


From this expression, we see that our analysis is valid only in the regime of energies for which $\eta^{2}>1 / 2$, which implies

$$
(E / \mathcal{M})^{\alpha}<1-\frac{1}{\sqrt{2}} \simeq 0.293
$$

This is not unreasonable, given that the concept of the effective field theory breaks down at the scale $\mathcal{M}$. Within this range of energies, one can justify self-consistently the approximation of ignoring terms of order $(E / \mathcal{M})^{2 \alpha}$ or higher.

We now compare the QG-modified spectrum (37) with that appearing in the standard Lorentz-invariant theory, namely:

$$
\frac{k^{2}-k_{0}^{2}-k_{3}^{2}}{4 \gamma}=\ell+s-\frac{1}{2}=\tilde{n}+\frac{1}{2}
$$

where $\tilde{n}=0,1,2, \ldots$ is the principal quantum number. In the relativistic case of interest to us, both the spectra (39) and (37) become quasi-continuous, since the quantum number $\tilde{n}$ becomes very large. QG corrections simply introduce a small rescaling (by a factor of order one) of the eigenvalues of the energy states which define the quantized orbiting radii.

Assuming that the motion is in the plane of the orbit $\left(k_{3}=0\right)$, one can then determine the radius $R$ of the orbit just by equating the energy eigenvalues as given by (37) with those obtained in the case of a particle in a magnetic field:

$$
c \hbar\left[4 \gamma\left(\frac{1}{2} \ell\left(\frac{\left(2 \eta^{2}-1\right)^{1 / 2}}{\eta}+1\right)+s \eta+\frac{\eta}{2}\right)\right]^{1 / 2}=e_{0} H R_{Q G},
$$

from which we obtain

$$
R_{Q G}=\left(\frac{\frac{1}{2} \ell\left(\frac{\left(2 \eta^{2}-1\right)^{1 / 2}}{\eta}+1\right)+s \eta+\frac{\eta}{2}}{\gamma}\right)^{1 / 2} .
$$

for the QG-modified radius $R_{Q G}$.

We are interested in macroscopic values of the orbit radius, which, in the Crab Nebula case discussed in $\left[12\right.$, lie in the range $\simeq 10^{10} \mathrm{~cm}$. Comparing this with the 
other scales in the problem, we see that we are in a situation where $\tilde{n}>>1$. Thus, the equation (40) can be approximated by

$$
\frac{R_{Q G}}{R_{0}} \approx \frac{1}{\sqrt{2}}\left(1+\sqrt{2-\frac{1}{\eta^{2}}}\right)^{1 / 2}
$$

where

$$
R_{0}=\left(\frac{\tilde{n}+\frac{1}{2}}{\gamma}\right)^{1 / 2}
$$

is the radius in the Lorentz-invariant theory.

Some comments about this expression are now in order. First, we remark on the functional dependence of $R_{0}$ on the principal quantum number $\tilde{n}$. In standard quantum mechanics, the mean square radius for a particle of spin $\mathrm{s}$ in a magnetic field is given by: $\bar{R}_{0}=\sum_{\xi} \int \Psi_{\tilde{n}, s, \xi}^{*} \Psi_{\tilde{n}, s, \xi} r d^{3} r$, where $\Psi_{\tilde{n} s}$ is the wave function, and the sum is over possible spin states $\xi$. In the case of the Klein-Gordon equation, $R_{0}$ is obtained by solving (30) exactly in the limit $\eta=1$, with the result

$$
\bar{R}_{0}^{K G}=\left(\frac{\tilde{n}}{\gamma}\right)^{1 / 2}\left(1+\frac{s+\frac{3}{2}}{4 \tilde{n}}\right) .
$$

On the other hand, in the case of a particle with spin $s=1 / 2$, corresponding to the Dirac equation in an external field, one obtains [25]:

$$
\bar{R}_{0}^{\text {Dirac }}=\left(\frac{\tilde{n}}{\gamma}\right)^{1 / 2}\left(1+\frac{s+\frac{1}{2}}{4 \tilde{n}}\right) .
$$

If one is interested in macroscopically large trajectories, as is the case for [12] and ourselves, where $\tilde{n} \gg 1$, we see from (44), (45) and (43) that all three formulae give approximately the same result. In particular, the spin dependence of the shape of the orbit is not significant. This justifies our initial simplification of working with a spinless particle instead of a Dirac fermion in order to estimate the QG modifications to the electron orbit.

This analysis demonstrates that QG modifies the critical synchrotron frequency (22) for radiation along the forward direction, in two ways. First by changing the relation of $\beta$ to energy, due to the $\mathrm{QG}$ modification of the dispersion relation, but 
also by modifying the orbit of the particle in the magnetic field, by slightly shrinking the average radius (42). Thus, we may parametrize the maximal frequency (6) as follows:

$$
\omega_{c}^{Q G}=\frac{3}{2} \frac{e H}{m_{0}} \frac{1}{1-\beta_{Q G}^{2}} \frac{R_{0}}{R_{Q G}},
$$

where the dependence on $R_{Q G}$ is dictated by the assumption of angular momentum conservation - the smaller the radius the higher the angular frequency. It is important to notice that, once one accepts the dispersion relation (17), the result (44) should be considered as exact for the entire range of energies for which (38) is valid, up to terms of higher order in $(E / \mathcal{M})^{\alpha}$.

Using the modified dispersion relation, then, identifying $\beta^{Q G}$ with the group velocity, and keeping only terms linear in $\left.(E / \mathcal{M})^{\alpha}\right)$, we obtain the following result for the group velocity:

$$
\begin{aligned}
& \beta_{Q G} \equiv \frac{\partial E}{\partial p}=\frac{p}{E}\left(1-\left(\frac{\alpha}{2}+1\right)\left(\frac{E}{\mathcal{M}}\right)^{\alpha}\right), \\
& 1-\beta_{Q G}^{2} \simeq \frac{m_{0}^{2}}{E^{2}}+(\alpha+1)\left(\frac{E}{\mathcal{M}}\right)^{\alpha} .
\end{aligned}
$$

On account of (42), then, this yields for the QG modification to the critical synchrotron radiation frequency:

$$
\omega_{c}^{Q G}=\frac{3}{\sqrt{2}} \frac{e H}{m_{0}} \frac{1}{\left(1+\sqrt{2-1 / \eta^{2}}\right)^{1 / 2}\left(\frac{m_{0}^{2}}{E^{2}}+(\alpha+1)\left(\frac{E}{\mathcal{M}}\right)^{\alpha}\right)} .
$$

This function is plotted schematically (for $\alpha=1$ ) in Fig. 1].

We observe that, since the effects on the radius are small for all values of $E / \mathcal{M}<0.3$, the discussion of [12] reviewed in the previous Section remains qualitatively correct. In particular, there is a global maximum within the range of energies allowed by (38), namely $\omega_{c, \max }^{Q G}$, which should be higher than the observed synchrotron energy of $0.5 \mathrm{GeV}$. The maximum occurs for electron energies $E_{\text {max }}=\left(2 m_{0}^{2} \mathcal{M}^{\alpha} / \alpha(\alpha+1)\right)^{1 /(2+\alpha)}$. From this, one may obtain bounds on $\alpha$, if one sets $\mathcal{M}=M_{P} \sim 10^{19} \mathrm{GeV}$, or, alternatively, obtain bounds for $\left|\xi_{e}\right|$ if one sets $\alpha$ to 


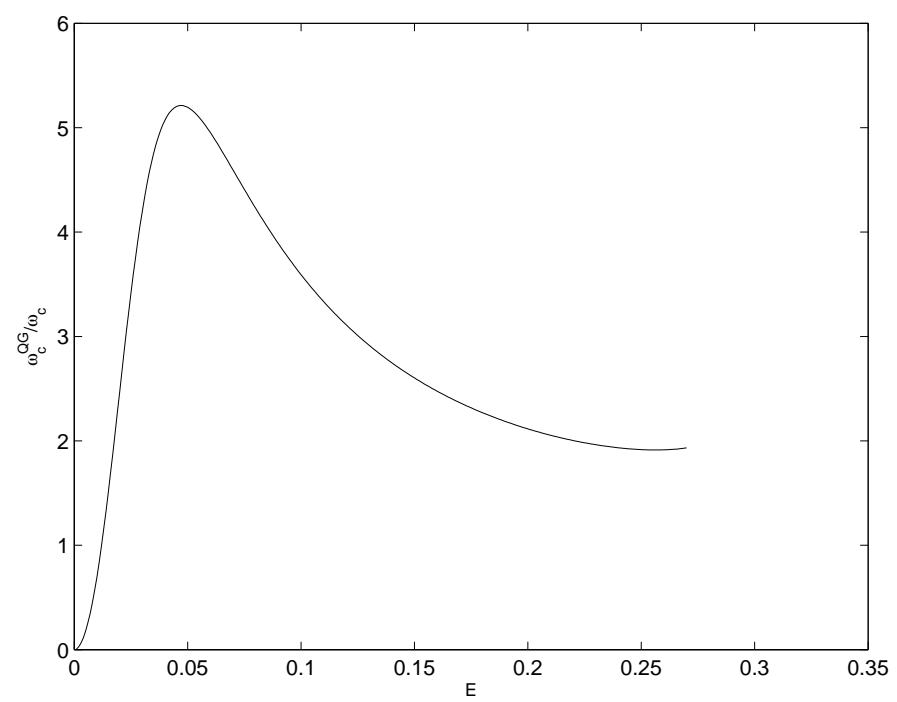

Figure 1: The scaled critical frequency for synchrotron radiation in quantum gravity: $\omega_{c}^{Q G} / \omega_{c}\left(\omega_{c}=3 e H / 2 m_{0}\right)$ as a function of the electron energy $E$ in units of $\mathcal{M}$, as obtained from (48) for $\alpha=1$. The synchrotron frequency is a bounded function of $E$, as in [12], a feature which is not affected by the QG modifications of the orbit.

a fixed value. It can be easily seen that the general formula (up to terms of order one) for the upper bound on $\left|\xi_{e}\right|$ is:

$$
\left|\xi_{e}\right|<\left(\frac{3 e H}{m_{0}}\right)^{\frac{\alpha+2}{2 \alpha}}\left(\frac{M_{P}}{m_{0}}\right)\left(\frac{2}{\alpha(\alpha+1)}\right)^{1 / \alpha}\left(\frac{\alpha}{\alpha+2}\right)^{(\alpha+2) / 2 \alpha} .
$$

If the resulting bound on $\left|\xi_{e}\right|$ is less than unity, the sensitivity exceeds the Planck scale for the given value of $\alpha$. For the linear case $\alpha=1$, the existence of the maximum implies that the discussion of [12] remains intact and one arrives at the bounds of $\xi_{e}$ given in (13), (14).

It is easy to see that for the average magnetic field of Crab Nebula ${ }^{7} H_{\text {cons }}=$

\footnotetext{
${ }^{7}$ It should be emphasized that the estimate of the end-point energy of the Crab synchrotron spectrum and of the magnetic field used above are indirect values based on the predictions of the Synchrotron Self-Compton (SSC) model of very-high-energy emission from Crab Nebula [27. We use a choice of parameters which gives good agreement between the experimental data on high-energy emission and the predictions of the SSC model [27, 28].
} 
$260 \mu \mathrm{G}\left[26\right.$ ] one obtains $\left|\xi_{e}\right|<6.8 \cdot 10^{21}\left(8.8 \cdot 10^{-21}\right)^{\frac{\alpha+2}{2 \alpha}}$, which implies that $\alpha \leq 1.72$ is excluded. If the lower value for $H_{n c o n s}=160 \mu \mathrm{G}\left[27\right.$ is used instead, then $\left|\xi_{e}\right| \leq 1$ for $\alpha \leq 1.74$; we also observe that for $\alpha=2\left|\xi_{e}\right|<\mathcal{O}(30-60)$ for the range of the magnetic field considered above. These imply already a sensitivity to quadratic QG corrections. Therefore,

$$
\alpha \geq \alpha_{c}: \quad 1.72<\alpha_{c}<1.74
$$

is the phenomenologically allowed range of $\alpha$, where the lower (upper) limit corresponds to $H_{\text {cons }}\left(H_{\text {ncons }}\right)$.

We should note that there are still some ambiguities regarding the magnitude of the maximal energy of synchrotron radiation before transition to inverse Compton emission, which could range from $30 \mathrm{MeV}^{8}$ to $0.5 \mathrm{GeV}$. This uncertainty should be resolved by future gamma-ray detectors such as GLAST [13, which could provide a better determination of the unpulsed gamma-ray spectrum in the energy range above $30 \mathrm{MeV}$, and thus a more precise determination of the maximum electron energy in the Crab Nebula.

Measurements of high-energy emissions from the Vela pulsar [29] indicate the operation of a mechanism for the production of very-high-energy gamma rays similar to that in Crab Nebula. This leads to an estimate of the magnetic field of order $H_{V e l a} \sim 3 \mu \mathrm{G}$. When used in (49), this yields $\alpha_{c}=2.04$, under the assumption that the first synchrotron hump is at the same location as in the Crab Nebula, namely $0.5 \mathrm{GeV}$. If, one the other hand, the hump is at $30 \mathrm{MeV}$, then $\alpha_{c}=1.86$. Therefore, improvements in measurements of synchrotron emission from the Vela Nebula (or other similar sources), which may be achieved in the not-too-distant future, could bring the sensitivity of such experiments closer to testing quadratic modifications of the electron dispersion relation.

This will be decisive for probing models with quadratic suppression of QG effects in the dispersion relation for electrons. However, we remind the reader that

\footnotetext{
${ }^{8}$ If the end-point of the Crab synchrotron spectrum is as low as $30 \mathrm{MeV}$, then the upper and lower limits for $\alpha_{c}$ in (50) become 1.56 and 1.58 respectively.
} 


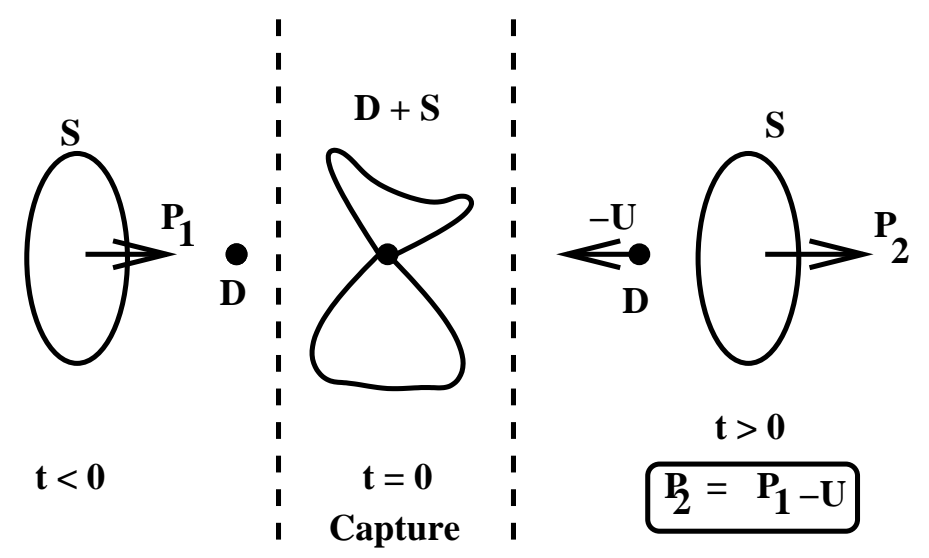

Figure 2: In the model of Liouville foam of [15], only string particles (S) neutral under the (unbroken) standard model group can be captured by the D-particle defects (D) in space time. This results in modified (subluminal) dispersion relations for $S$, as a consequence of the recoil of the defect $D$ in a direction opposite to the incident beam, after the capture stage.

synchrotron radiation provides no significant bounds for QG modifications to the dispersion relation for photons [12].

\section{Violations of the Equivalence Principle in the Liouville Model for Space-Time Foam}

As already mentioned, the synchrotron radiation constraint [12] on the dispersion relation for electrons, refined in the previous Section, does not exclude the possibility of a larger QG modification of the dispersion relation for photons. Of course, this would require the violation of the equivalence principle for energetic particles. Remarkably, this is exactly what is predicted by our Liouville string/D-particle model for space-time foam, according to which only gauge bosons such as photons might have QG-modified dispersion relations, and not charged matter particles such as electrons.

This difference may be traced to a cornerstone of D-brane physics, namely that 
excitations which are charged under the gauge group are represented by open strings with their ends attached to the D-brane [16], and only neutral excitations are allowed to propagate in the bulk space transverse to the brane.

Our D-particle model of QG foam is based on point-like defects in space-time, that are nothing but zero-space-dimensional D-branes, embedded in a four- (or higher-) dimensional bulk Minkowski space-time. These affect the propagation of closed-string states via a recoil process [15] (see Fig. 21), that is associated with back-reaction effects in target space, which lead to the QG-modified effective metric (18). In this picture, a closed-string state propagating in the bulk can be captured by the defect and split into two open-string excitations with their ends attached to the defect, which later recombine to become a closed-string state. According to the above-mentioned property of D-branes, the open-string excitations of the D-particle defect result in massless $U(1)$ excitations for a single defect, or massless gauge bosons in the adjoint representation of $\mathrm{U}(\mathrm{N})$ for a group of coincident recoiling D-particles [30, 31].

In the original formulation of this D-particle model of QG foam [15], the Dparticles have no 'hair', i.e., they have only vacuum quantum numbers. Therefore, they can absorb closed-string states only if they also carry no conserved charges. This would exclude any QG medium effects on particles with electromagnetic or colour charge, certainly including electrons and quarks. On the other hand, a QG effect would be expected for the photon, which carries no conserved charge. Because the electron has no interaction with the QG vacuum medium in this approach, it emits no Čerenkov radiation, despite traveling faster than photons, thus avoiding the vacuum Čerenkov radiation constraint considered by [32].

Neutrinos are mainly doublets of the SU(2) subgroup of the Standard Model. However, in generic seesaw models of neutrino masses, the light neutrinos mix with $\mathcal{O}\left(m_{W} / M\right)$ components of gauge-singlet states, perhaps opening the window for QG effects, depending on details of the model. However, any such effects would be suppressed by at least a similar factor. In the case of gluons, which carry nonAbelian charges, it is possible that ensembles of D-particle defects would have a QG 
effect on their dispersion relations, which would in general be unrelated to that on the photon.

However, such a relation might appear if the $\mathrm{U}(1)$ of electromagnetism is actually embedded in some simple GUT group such as SU(5) 33, so that the photon becomes some combination of non-Abelian gauge fields, in which case QG effects on its dispersion relation might be related to those of the gluons. On the other hand, there exist non-simple GUT models, such as flipped $\mathrm{SU}(5) \times \mathrm{U}(1)$ [34], in which the photon contains a significant component from outside the non-Abelian group factor, in which case the photon's dispersion relation will in general differ from those of the gluons.

Even if the dispersion relations of individual gluons are modified by QG effects, these may be suppressed for colour-singlet hadrons such as protons. A QG modification of the proton's dispersion relation has been suggested as a way of explaining the possible existence of UHECRs beyond the GZK cut-off [10], where energy nonconservation in high-energy reactions might also become significant [18, 19]. As observed in [19], in the case of a linear QG modification of the proton's dispersion relation similar to $(2)$, the magnitude of the analogous parameter $\xi_{p}$ would need to be of order $10^{-16}-10^{-17}$. Such a suppression is (coincidentally?) $\mathcal{O}\left(\Lambda_{Q C D} / M_{P}\right)$, and such a suppression cannot be excluded at present, though a lot more work is required before such a conclusion could be reached in the framework of the D-particle model of 15. However, the above discussion makes it clear that the details of the dynamics of the interactions between particles and space-time foam are potentially important, and any naive phenomenological assumption of the equivalence principle could be misleading.

Before closing, we comment briefly on recent claims that modified dispersion relations for photons would result in phase incoherence of light from distant galaxies 35]. Based on this suggestion, these authors proposed a stellar interferometry technique, and claimed to place stringent bounds on the effective quantum-gravity scale for photons, excluding linearly-modified dispersions. However, their arguments have been criticized in [36], on the basis that they overestimated the induced inco- 
herent effects by a large factor $(L / \lambda)^{\alpha}$, where $L$ is the distance of the source, $\lambda$ is the photon wavelength, and $\alpha$ is the parameter in the modified dispersion relation of the photon probe. The correct amount of cumulative phase incoherence induced by quantum gravity is [36]: $\delta \phi \sim(L / \lambda)^{1-\alpha}$. Thus, for linear dispersion relations for photons $(\alpha=1)$ such as those proposed in [8, 15], the technique of [35] cannot be used. Moreover, in the case of the D-particle recoil model for Liouville foam [15] the re-emission of the photon from the recoiling D-particle (c.f. Fig. 2) is accompanied by a (random) phase in the photon's wave-function. This would destroy any cumulative phase incoherence, in contrast to the claims in 35]. Thus, a linear modification of the photon dispersion relation cannot be excluded by this argument.

Our analysis emphasizes the interest in probing independently the dispersion relation of the photon. The study of the arrival times of photons from gamma-ray bursts 9] still appears to be the best experimental probe of any possible refractive index for photons, and should be pursued further in the future.

The mechanism for violating the equivalence principle discussed in this article may not be the only route to consistency with the current experimental constraints. However, it is certainly one promising way, in the sense that it does not invoke exceedingly small parameters, and appears naturally within a class of stringy models of quantum gravity [15]. In other approaches to quantum gravity, such as loop canonical quantum gravity [4, one may encounter models which feature linear modifications in the dispersion relations for matter excitations that are characterized by extra small parameters which can be bound by experiments. Such a scenario has been discussed recently in [37, where the structure of canonical (quantum) commutators between momenta and position operators in loop-gravity models is modified by extra scaling terms, described by a set of coefficients that are essentially free parameters of the model. Such extra terms lead to linear modifications in dispersion relations, but with coefficients that are proportional to these parameters. The latter can then be bounded by requiring consistency of the models with the current experimental situation. In our opinion, the disadvantage of such an approach lies in the unnaturally small values one obtains for such parameters in this case, which is to be 
contrasted with our case above, where the numerical coefficient in front of the linear modification of the photon dispersion relation is naturally of order one. However, we cannot exclude the possibility this, or some other way yet to be invented, may be the way that Nature evades the current experimental constraints on quantum gravity.

\section{Acknowledgements}

We would like to thank H. Hofer for his interest and support. The work of N.E.M. is partially supported by a visiting professorship at the University of Valencia (Spain), Department of Theoretical Physics, and by the European Union (contract HPRNCT-2000-00152).

\section{References}

[1] G. Amelino-Camelia, J. R. Ellis, N. E. Mavromatos and D. V. Nanopoulos, Int. J. Mod. Phys. A 12 (1997) 607 arXiv:hep-th/9605211.

[2] J. R. Ellis, N. E. Mavromatos and D. V. Nanopoulos, Phys. Lett. B 293 (1992) 37 arXiv:hep-th/9207103; For reviews see: J. R. Ellis, N. E. Mavromatos and D. V. Nanopoulos, Erice Subnucl. Phys. Series, Vol. 311 (World Sci. 1994) arXiv:hep-th/9311148; J. Chaos, Solitons and Fractals, Vol. 10 (1999) 345 (eds. C. Castro amd M.S. El Naschie, Elsevier Science, Pergamon 1999) arXiv:hep-th/9805120.

[3] L. Gonzalez-Mestres, arXiv:physics/9703020, arXiv:physics/9712056.

[4] R. Gambini and J. Pullin, Phys. Rev. D 59 (1999) 124021 arXiv:gr-qc/9809038; For a review see: L. Smolin, arXiv:hep-th/0303185 and references therein. 
[5] G. Amelino-Camelia, Int. J. Mod. Phys. D 11 (2002) 35 arXiv:gr-qc/0012051; J. Magueijo and L. Smolin, Phys. Rev. Lett. 88 (2002) 190403 arXiv:hep-th/0112090.

[6] S. R. Coleman and S. L. Glashow, Phys. Lett. B 405 (1997) 249 arXiv:hep-ph/9703240; arXiv:hep-ph/9808446.

[7] C. P. Burgess, J. Cline, E. Filotas, J. Matias and G. D. Moore, JHEP 0203 (2002) 043 arXiv:hep-ph/0201082.

[8] G. Amelino-Camelia, J. R. Ellis, N. E. Mavromatos, D. V. Nanopoulos and S. Sarkar, Nature 393 (1998) 763 arXiv:astro-ph/9712103.

[9] J. R. Ellis, K. Farakos, N. E. Mavromatos, V. A. Mitsou and D. V. Nanopoulos, Astrophys. J. 535, 139 (2000) arXiv:astro-ph/9907340; J. R. Ellis, N. E. Mavromatos, D. V. Nanopoulos and A. S. Sakharov, Astron. Astrophys. 402, 409 (2003) arXiv:astro-ph/0210124.

[10] G. Amelino-Camelia and T. Piran, Phys. Rev. D 64 (2001) 036005 arXiv:astro-ph/0008107.

[11] D. Sudarsky, L. Urrutia and H. Vucetich, Phys. Rev. Lett. 89 (2002) 231301 arXiv:gr-qc/0204027; J. Alfaro, H. A. Morales-Tecotl and L. F. Urrutia, Phys. Rev. D 66 (2002) 124006 arXiv:hep-th/0208192 . R. C. Myers and M. Pospelov, Phys. Rev. Lett. 90 (2003) 211601 arXiv:hep-ph/0301124.

[12] T. Jacobson, S. Liberati and D. Mattingly, Nature 424 (2003) 1019 arXiv:astro-ph/0212190.

[13] F. W. Stecker, arXiv:astro-ph/0304527 and references therein.

[14] S. D. Biller et al., Phys. Rev. Lett. 83 (1999) 2108 arXiv:gr-qc/9810044;

P. Kaaret, arXiv:astro-ph/9903464 
[15] J. R. Ellis, N. E. Mavromatos and D. V. Nanopoulos, Phys. Rev. D 61 (2000) 027503 arXiv:gr-qc/9906029; Phys. Rev. D 62 (2000) 084019 arXiv:gr-qc/0006004.

[16] J. Polchinski, TASI Lectures on D-branes, arXiv:hep-th/9611050.

[17] J. R. Ellis, N. E. Mavromatos, D. V. Nanopoulos and A. S. Sakharov, arXiv:gr-qc/0312044.

[18] Y. J. Ng, D. S. Lee, M. C. Oh and H. van Dam, Phys. Lett. B 507 (2001) 236 arXiv:hep-ph/0010152.

[19] J. R. Ellis, N. E. Mavromatos and D. V. Nanopoulos, Phys. Rev. D 63 (2001) 124025 arXiv:hep-th/0012216.

[20] G. D. Moore and A. E. Nelson, JHEP 0109 (2001) 023 arXiv:hep-ph/0106220.

[21] G. Amelino-Camelia, arXiv:gr-qc/0212002.

[22] T. Jacobson, S. Liberati and D. Mattingly, arXiv:gr-qc/0303001.

[23] S. R. Coleman and S. L. Glashow, Phys. Rev. D 59 (1999) 116008 arXiv:hep-ph/9812418.

[24] J. R. Ellis, N. E. Mavromatos, D. V. Nanopoulos and G. Volkov, Gen. Rel. Grav. 32 (2000) 1777 arXiv:gr-qc/9911055.

[25] A. A. Sokolov and I. M. Ternov, "Radiation From Relativistic Electrons,", edited by C. W. Kilmister, AIP translation Series (1986).

[26] O.C. de Jager et al., Astrophys. J. 457 (1996) 253.

[27] R. A. Ong, Phys. Rept. 305 (1998) 93.

[28] A. M. Hillas et al., Astrophys. J. 503 (1998) 744.

[29] T. Yoshikoshi et al., Astrophys. J. 487 (1997) L65. 
[30] E. Witten, Nucl. Phys. B460 (1996) 335.

[31] N. E. Mavromatos and R. J. Szabo, Phys. Rev. D 59 (1999) 104018 arXiv:hep-th/9808124.

[32] S. Liberati, T. A. Jacobson and D. Mattingly, arXiv:hep-ph/0110094; T. Jacobson, S. Liberati and D. Mattingly, Phys. Rev. D 66 (2002) 081302 arXiv:hep-ph/0112207; T. J. Konopka and S. A. Major, New J. Phys. 4 (2002) 57 arXiv:hep-ph/0201184; T. Jacobson, S. Liberati and D. Mattingly, Phys. Rev. D 67 (2003) 124011 arXiv:hep-ph/0209264.

[33] H. Georgi and S. L. Glashow, Phys. Rev. Lett. 32 (1974) 438.

[34] I. Antoniadis, J. R. Ellis, J. S. Hagelin and D. V. Nanopoulos, Phys. Lett. B 194 (1987) 231; Phys. Lett. B 205 (1988) 459 and Phys. Lett. B 231 (1989) 65.

[35] R. Lieu and L. W. Hillman, arXiv:astro-ph/0211402; R. Ragazzoni, M. Turatto and W. Gaessler, Astrophys. J. 587 (2003) L1 arXiv:astro-ph/0303043.

[36] Y. J. Ng, H. van Dam and W. A. Christiansen, Astrophys. J. 591 (2003) L87 arXiv:astro-ph/0302372; Y. J. Ng, Mod. Phys. Lett. A 18 (2003) 1073 arXiv:gr-qc/0305019.

[37] G. Dotti and R. J. Gleiser, Phys. Rev. D $67 \quad$ (2003) 101501 arXiv:gr-qc/0302086. 